\title{
Clinical and anatomical features of the central part of the retina in diabetic macular edema
}

\begin{abstract}
Purpose: To study diagnostic criterion of the pathological process in patients with diabetic macular edema (DME): morphological changes of the retinal layers, light sensitivity, macular pigment optical density.
\end{abstract}

Methods: In this study 58 patients were divided in two groups. The first group (A) include 32 patients with DME (36 eyes), the second group (B) -26 patients (38 eyes) were without retinal pathology. Parameter estimation was conducted with the use of following methods: by optical coherence tomography (OCT) of macular area of the retina; photo-registration fundus with the measurement of the optical density of macular pigment (MPOD); microperimetry.

Results: OCT analysis showed that the inner nuclear and plexiform layers of retina were the most involved in pathological process and revealed significant differences in increase of mean retinal thickness in comparison with the control group. Analysis of MPOD revealed a significant reduction of all parameters in group A compared with the control group. On the basis of microperimetry of the central area of retina in group a light sensitivity indicators were reduced by an average of $10 \mathrm{~dB}$ and equaled $8.94 \pm 0.09 \mathrm{~dB}$. Correlation analysis of retinal thickness, functional light sensitivity and optical density of macular pigment traced a strong connection $(r=-0.94 ; p<0.05)$ between the indices of retinal thickness and MPOD.

Conclusion: Showed a sharp decrease in optical density of macular pigment determined by deficiency of lutein and zeaxanthin in patients with DME. A correlation between quantitative indicator of MPOD and area of edema was established.

Keywords: diabetic macular edema, macular pigment optical density, optical coherence tomography, microperimetry

\author{
Volume 5 Issue I - 2016
}

\author{
Bikbov Mukharram Mukhtaramovich, \\ Fayzrakhmanov Rinat Rustamovich, Rinat \\ Zainullin Mukhametovich \\ Department of Ophthalmology, Ufa Eye Research Institute, \\ Russia
}

Correspondence: Rinat Zainullin Mukhametovich, Ufa Eye Research Institute, Pushkin str. 90, Ufa, 450008, Russia, Tel +7 (965) 928-54-60; +7 (347) 255-57-17

Email rinatmedical@mail.ru

Received: September 05, 2016 | Published: October 5, 2016
Abbreviations: DME, diabetic macular edema; OCT, optical coherence tomography; MPOD, measurement of the optical density of macular pigment

\section{Introduction}

One of the most significant manifestations of diabetes is retinal damage in the form of diabetic macular edema (DME), leading to inevitable loss of vision and disability of patients of the working age. ${ }^{1}$ Early diagnostics allows identifying the disease at an early stage of development and reducing the risk of irreversible loss of vision. Currently, one of the main ways of retinal lesions visualization ${ }^{2}$ and DME is optical coherence tomography (OCT) enabling to assess the thickness and structure of the retina. ${ }^{3-5}$ Several authors established that a macular pigment characterizing the state of the outer layers of the retina and, in particular, the state of photoreceptors has a direct effect on the light sensitivity of the retina. ${ }^{6}$ Regular and objective measurement of macular pigment optical density (MPOD) is justified method of research in pathology of the central part of the retina. ${ }^{7}$

However, in references there are only a few reports on distribution of macular pigment and its effect on functional characteristics at DME. ${ }^{8}$ One of the most informative methods of assessment of the macular area functional state is microperimetry, which allows defining areas of macular light sensitivity even in patients with low visual acuity. During the study the data superimposition on a colour picture of the fundus is made, which enables to determine accurately functional properties of different parts of the pathological focus. ${ }^{9}, 10$ Thus, defining the macular pigment density and its correlation with functional and morphological parameters of the central part of the retina in DME can serve as an additional diagnostic criterion for assessing the pathological process nature. Estimation of morphological changes in the retinal layers and macular pigment optical density can be used as diagnostic criteria to assess the pathological process in patients with diabetic macular edema.

\section{Materials and methods}

A written informed consent for participating in the study was obtained from all patients, undergoing the examination. The study was approved by the Ufa Eye Research Institute ethics committee (July 7, 2014 (№ 3) protocol) and was performed according to tenets of the Declaration of Helsinki. Thirty two patients (38 eyes) with newly diagnosed diabetic macular edema, observed in 2014, were selected by random sampling technique. Patients had not received special eye treatment (laser macular treatment, injections, etc.) by the time of the examination. Patients with concomitant diseases of the retina were not included in the study. Patients with diabetic macular edema (36 eyes) were included in Group I (study), 26 patients with no retinal pathology (38 eyes) were included in Group II (control). The demographic and clinical characteristics of these patients are presented in (Table 1). Only those patients who had a definite diagnosis of DME and nonproliferative diabetic retinopathy were included.

All the patients with other retinopathies mimicking DME, or with any other retinal lesion or any other factor (e.g., cataract) including 
any treatment for DME were excluded. Standard ophthalmic examination included ophthalmobiomicroscopy with the usage of aspheric lens $78 \mathrm{D}$, OCT of macular region by tomography (Retina Scan, RS-3000, NIDEK, Gamagori, Japan), Macular Multi Cross mode. The fundus photographic recording with MPOD measurement via auto fluorescence spectrometry using a non mydriatic fundus camera VISUCAM 500® (Carl Zeiss Meditec AG, Jena, Germany), microperimetry (MP1 Microperimeter, Nidek Technologies, Italy). In calculating OCT structural values a retinal area of 6,000 micron with a centre in foveola of each layered zone was used. The total thickness of the retina and thickness of each layer were analyzed in increments of 300 micron in 21 points (Table 1).

Table I Demographic and clinical characteristics

\begin{tabular}{|c|c|c|}
\hline Demographic Variable & Group I (Study) & Group II (Control) \\
\hline \multicolumn{3}{|l|}{ Gender } \\
\hline Male & $14-43.70 \%$ & II (42.3\%) \\
\hline Female & $18-56.30 \%$ & I 5 (27.7 \%) \\
\hline \multicolumn{3}{|l|}{ Age at Visit } \\
\hline Mean $\pm S D$ & $53.5 \pm 3.4$ & $52.5 \pm 2,8$ \\
\hline Range & $41-66$ & $38-67$ \\
\hline \multicolumn{3}{|c|}{ Distribution by Age (Count) } \\
\hline$\%)<45$ & 9 (28.1 \%) & $8(32.0 \%)$ \\
\hline $45-<65$ & $18-56.30 \%$ & $13(52.0 \%)$ \\
\hline$\geq 65$ & $5(15.6 \%)$ & $4(16.0 \%)$ \\
\hline \multicolumn{3}{|l|}{ Eye Involvement } \\
\hline Right eye & 18 (56.25 \%) & II (44.0\%) \\
\hline Left eye & $12-37.50 \%$ & $8(32.0 \%)$ \\
\hline Both eyes & $2-6.20 \%$ & 6 (24.0\%) \\
\hline
\end{tabular}

Using parameters of the fundus camera (Volume, Area, Max, Mean) in a MPOD mode, MPOD was defined in Groups I and II of patients. The auto fluorescence of the fundus at differing wavelengths was captured on video frames. Digital subtraction of the reflectance maps at 488 and $514 \mathrm{~nm}$, with adjustments made for absorption by the lens, yielded the mean macular pigment density. ${ }^{7}$ During the microperimetry a reference stimulus of $0,43^{\circ}$ size (Goldmann III) and 200-ms duration was applied. An automatic program testing 45 points of twelve degrees with a centre in the fixation area was applied; the study was carried out with an accuracy of $2 \mathrm{~dB}$. Localization of the point of gaze fixation and its stability over time was determined by a fixation test. Statistical analysis was performed using methods of descriptive statistics, one-way ANOVA test and Duncan's test for multiple comparisons. Differences were considered significant at $p$ $<0,05$. The results of descriptive statistics in most of the tables are presented in the form of $\mathrm{M} \pm \mathrm{y}$, where $\mathrm{M}$-mean value, $\mathrm{y}$-standard deviation. The critical level of statistical significance when testing the null hypothesis was set at 0.05 .

\section{Results}

Changes typical of diabetic retinopathy were observed ophthalmoscopically in patients of Group I in the form of microaneurysms, "hard" and/or "soft" exudates, and retinal edema, intraretinal microvascular abnormalities (pronounced veins, unevenness of their diameter, tortuosity, and vascular loops). According to the classification by ETDRS, patients in this group had mild and moderate nonproliferative diabetic retinopathy. On Table 2 shows the study results of retinal morphological structures in patients of compared groups. In the analysis of OCT cartograms in patients of II Group a correct macular profile with a fovea in the centre was determined. Coverage of total retinal thickness ranged from $220,2 \pm 11,92$ to $343,4 \pm 10,14$ micron. The mean value was equal to $298,3 \pm 6,43$ micron. Patients in Group I had the mean retinal thickness taking into account all points of the study area from $340,43 \pm 36,04$ to $463,18 \pm 38,21$ micron, with a gradual thickening from the periphery towards the centre. The maximum values were observed at a distance of 889 micron from the centre of the fovea. The mean value of retinal thickness as a result of edema increased by $35 \%$ and amounted to 404,62 $\pm 15,23$ micron.

Table 2 Mean parameters of retinal thickness in patients of compared groups, micron

\begin{tabular}{llll}
\hline & Layered areas of the retina & Group I & Group II \\
\hline Areas & Total thickness & $404.62 \pm 15.23^{* *}$ & $298.3 \pm 6.43$ \\
\hline & $\begin{array}{l}\text { Retinal pigment epithelium- } \\
\text { outer segments of } \\
\text { Photoreceptors }\end{array}$ & $63.26 \pm 1.01$ & $71.14 \pm 0.78$ \\
& $\begin{array}{l}\text { Inner segments of } \\
\text { photoreceptors-Outer } \\
\text { Nuclear layer }\end{array}$ & $87.38 \pm 3.85$ & $75.35 \pm 3.06$ \\
3 & $\begin{array}{l}\text { Outer plexiform layer-inner } \\
\text { nuclear layer }\end{array}$ & $114.97 \pm 4.92^{* *}$ & $61.09 \pm 3.96$ \\
4 & $\begin{array}{l}\text { Inner plexiform layer-Ganglion } \\
\text { cell layer }\end{array}$ & $109.87 \pm 4.86^{*}$ & $68.46 \pm 3.95$ \\
5 & $\begin{array}{l}\text { Nerve fiber layer-inner limiting } \\
\text { membrane }\end{array}$ & $28.67 \pm 2.21^{*}$ & $12.65 \pm 1.2$ \\
\hline
\end{tabular}

*Significant differences compared to the control group $(p<0.05)^{* *}$ - $p<0.00$ I

Zonal analysis showed that the mean value of thickness of the retinal first zone in patients of the Group I decreased by $16 \%$ due to the swollen component of the overlying layer. Values of the second zone showed a $15 \%$ increase indicating a partial involvement of these structures. The greatest changes were marked in the 3, 4 and 5 areas where the mean increase in retinal thickness as compared to the control group was significant. While interpreting the results of MPOD studies, graphic cartograms as profiles of spatial distribution of the macular pigment, and the measured values (average optical density, maximum optical density, volume and area) were analyzed. When analyzing MPOD significant reduction of all parameters in patients of the group I was detected compared with the control group (Table 3 ). It was revealed a marked reduction in the optical density of the macular pigment in the area of retinal edema when comparing OCT and MPOD data. Based on the microperimetry data of the central zone of the retina in patients of the group I the gaze fixation remained central and stable, and the size of the field of fixation did not exceed $4^{\circ}$. Parameters of light sensitivity were reduced by an average of $10 \mathrm{~dB}$ and amounted to $8,94 \pm 0,09 \mathrm{~dB}$. In case of stimuli hitting the area of hard exudates a marked reduction in sensitivity was observed $(0 \mathrm{~dB})$ compared to the area of macular edema (up to $4 \mathrm{~dB}$ ). 
Table 3 MPOD mean values in patients of the Groups I and II

\begin{tabular}{lllll}
\hline & Max OD (du) & Mean OD (du) & Area (Pixel) & Volume (du x pixel) \\
\hline Group I & $0.321 \pm 0.01 *$ & $0.102 \pm 0.01 *$ & $54484.92 \pm \mid 4769.36$ & $4106.83 \pm 1080.58^{*}$ \\
Group II & $0.693 \pm 0.02$ & $0.252 \pm 0.01$ & $63651 \pm 15234.23$ & $15702 \pm 3043.23$
\end{tabular}

*Significant differences compared to the control group $(\mathrm{p}<0.05)$. du = Density Units; OD = Optical Density

Thus, analysis of the data revealed gross disruption of the structure and distribution of MPOD in patients with DME. Inner nuclear and inner plexiform layer of the retina proved to be the most involved in the pathological process. Correlation analysis of morphological (retinal thickness), functional parameters (light sensitivity), and optical density of the pigment epithelium showed strong inverse correlation $(\mathrm{r}=-0,93 ; \mathrm{p}<0,05)$ between parameters of retinal thickness and macular pigment optical density. According to the study, considering the correlation of MPOD and photoreceptors as well as decrease in density of the macular pigment in the areas of edema, the involvement of the photoreceptor layer in the pathological process is quite possible. The issue of the secondary destruction of photoreceptors in DME requires further detailed study with additional research methods. Changes marked over time can be used as a parameter for determining the disease prognosis. The MPOD studying may be a form of DME patients screening, comparable to the visual field examination or measurement of the nerve fiber layer thickness in glaucoma.

\section{Discussion}

In patients with diabetes mellitus that is lasting for 20 years or more visual disorders caused by macular edema occur in $29 \%$ of cases. ${ }^{11}$ These changes include a wide range of pathological processes leading to retina thickening due to formation of macular edema and appearance of hard exudates. Conventional diagnostic methods of retinal diseases such as biomicroscopy, indirect ophthalmoscopy and stereo fundus photography are not always informative to detect small changes in retinal thickness. ${ }^{12,13}$ Gieser et al., ${ }^{14}$ (1997) were the first who described retina structure and gave an objective assessment of its thickness according to the OCT. According to the group of authors ${ }^{14-16}$ there is a significant correlation between the average thickness of macular area (OCT data) and visual acuity in patients with diabetes and diabetic retinopathy in laser and/or intravitreal therapy over time. ${ }^{17-19}$ However, other studies have shown no correlation or its presence in a moderate degree and there is still no consensus on this matter. $^{20}$

Yohannan et al., ${ }^{21}$ report a correlation between a sensitivity of retina and photoreceptor state in patients with diabetic macular edema (DME). This study points out that disorder in internal segments of photoreceptors is correlated with a significant reduction of eyes sensitivity with DME. In addition, the study by Querques et al., ${ }^{22}$ reports that a decrease in sensitivity of the retina is consistently correlated with morphological abnormality in inner segments of photoreceptors in patients with retina diseases. Research by Reibaldi et al., ${ }^{23}$ informs that preserving the outer limiting membrane contributes to the maintenance of visual acuity in patients with lamellar macular rupture. According to Fujita et al., ${ }^{24}$ in patients with central serous chorioretinopathy undergoing photodynamic therapy, improvement of vision correlates with recovery of the photoreceptor layer after
6 and 12 months after therapy. Each of these studies suggests that the state of the outer layers of the retina is strongly associated with functional component of vision. The results of our study show that microperimetry results worsen at involvement of mainly inner retinal layers in the pathological process. Thus, the microperimetry data are sensitive and useful for assessing functional disorders in the inner layers of the retina.

In contrast to other studies, which evaluate the ratio between the thickness of the central retina and light sensitivity, this study provides data showing the correlation between the thickness of the retina and the corresponding microperimetric data in the foveal and parafoveal region. According to our records, there was a reduction of macular pigment density level, which may be caused by increased oxidative stress. ${ }^{25}$ Macular pigment is likely to play an important role in the homeostasis of the retina when exposed to oxidative stress. The absence of significant density of macular pigment along with oxidative damage in diabetes can contribute significantly to the pathogenesis of DME. In the course of our investigation a correlation between a quantitative indicator of macular pigment optical density and the area of retinal edema is established. These results have no counterparts in other studies. It should be determined whether local or diffuse defects of macular pigment density increase the risk of further oxidative damage and therefore the progression of DME. Therefore, large-scale research is necessary in patients with DME, with a focus on the disease duration and results of therapy to be correlated with changes in the density of macular pigment and its distribution, visual acuity, and the results of other retina research methods.

\section{Conclusion}

A sharp decline in macular pigment optical density determined by its scarcity was observed in diabetic macular edema. A negative correlation between a quantitative parameter of macular pigment optical density and the area of retinal edema was established. Light sensitivity in the central area of the retina was reduced significantly. When stimuli were projected in the area corresponding to the zone of hard exudates deposition, a severe abnormality of the light sensitivity to the extent of its absence was revealed.

\section{Acknowledgments}

None.

\section{Conflicts of interest}

Authors declare that there is no conflict of interest.

\section{References}

1. Chang CK, Cheng CK, Bai CH, et al. Development of vitreomacular interface abnormality in patients with diabetic macular edema. Taiwan Journal of Ophthalmology. 2012;2(3):93-98. 
2. Nisic F, Turkovic S, Mavija M, et al. Correlation Between the Findings of Optical Coherent Retinal Tomography (OCT), Stereo Biomicroscopic Images from Fundus of an Eye and Values from Visual Acuity of Diabetic Macular Edema. Acta Inform Med. 2014;2:232-236.

3. Jackson TL, Nicod E, Angelis A, et al. Vitreous attachment in agerelated macular degeneration, diabetic macular edema, and retinal vein occlusion: a systematic review and metaanalysis. Retina. 2013;33:1099-1108.

4. Varano M, Scassa C, Ripandelli G, et al. New diagnostic tools for macular edema. Doc Ophthalmol. 1999;97(3-4):373-379.

5. Hodzic-Hadzibegovic D, Sander BA, Lund-Andersen H. Diabetic macular oedema quantified with spectral-domain optical coherence tomography-evaluation of boundary line artefacts and the effect on retinal thickness. Acta Ophthalmol. 2015;93(1):74-82.

6. Lardenoye CW, Probst K, DeLint PJ, et al. Photoreceptor function in eyes with macular edema. Invest Ophthalmol Vis Sci. 2000;41(12):4048-4053.

7. Rothenbuehler SP, Wolf-Schnurrbusch UEK, Wolf S. Macular pigment density at the site of altered fundus autofluorescence. Graefes Arch Clin Exp Ophthalmol. 2011;249(4):499-504.

8. Waldstein SM, Hickey D, Mahmud I, et al. Two-wavelength fundus autofluorescence and macular pigment optical density imaging in diabetic macular oedema. Eye (Lond). 2012;26(8):1078-1085.

9. Midena E, Radin PP, Pilotto E, et al. Fixation pattern and macular sensitivity in eyes with subfoveal choroidal neovascularization secondary to age-related macular degeneration. A microperimetry study. Semin Ophthalmol. 2004;19(1-2):55-61.

10. Querques G, Forte R, Longo C, et al. Microperimetry in age-related macular degeneration. J Fr Ophtalmol. 2008;31(5):515-521.

11. Klein R, Klein BE, Moss SE, et al. The Wisconsin epidemiologic study of diabetic retinopathy. IV. Diabetic macular edema. Ophthalmology. 1984;91(12): 1464-1474.

12. Otani T, Kishi S, Maruyama Y. Patterns of diabetic macular edema with optical coherence tomography. Am J Ophthalmol. 1999;127(6):688-693.

13. Mylonas G, Bolz M, Kriechbaum K, et al. Retinal architecture recovery after grid photocoagulation in diabetic macular edema observed in vivo by spectral domain optical coherence tomography. Retina. 2013;33(4):717-725.

14. Gieser JP, Rusin MM, Mori M, et al. Clinical assessment of the macula by retinal topography and thickness mapping. Am J Ophthalmol. 1997;124(5):648-660.
15. Martidis A, Duker JS, Greenberg PB, et al. Intravitreal triamcinolone for refractory diabetic macular edema. Ophthalmology. 2002;109(5):920-927.

16. Jonas JB, Kreissig I, Söfker A, et al. Intravitreal injection of triamcinolone for diffuse diabetic macular edema. Arch Ophthalmol. 2003;121(1):57-61.

17. Hannouche RZ, Avila MP, Isaac DL, et al. Correlation between central subfield thickness, visual acuity and structural changes in diabetic macular edema. Arq Bras Oftalmol. 2012;75(3):183-187.

18. Hannouche RZ, Avila MP, Isaac DL, et al. Correlation between central subfield thickness, visual acuity and structural changes in diabetic macular edema. Arq Bras Oftalmol. 2012;75(3):183-187.

19. Shimura M, Yasuda K, Yasuda M, et al. Visual outcome after intravitreal bevacizumab depends on the optical coherence tomographic patterns of patients with diffuse diabetic macular edema. Retina. 2013;33(4):740-747.

20. Ito S, Miyamoto N, Ishida $\mathrm{K}$, et al. Association between external limiting membrane status and visual acuity in diabetic macular oedema. Br J Ophthalmol. 2013;97(2):228-232.

21. Yohannan J, Bittencourt M, Sepah YJ, et al. Association of retinal sensitivity to integrity of photoreceptor inner/outer segment junction in patients with diabetic macular edema. Ophthalmology. 2013;120(6):1254-1261.

22. Querques L, Querques G, Forte R, et al. Microperimetric correlations of autofluorescence and optical coherence tomography imaging in dry age-related macular degeneration. Am J Ophthalmol. 2012;153(6):1110-1115.

23. Reibaldi M, Parravano M, Varano M, et al. Foveal microstructure and functional parameters in lamellar macular hole. Am J Ophthalmol. 2012;154(6):974-980.

24. Fujita K, Shinoda K, Imamura Y, et al. Correlation of integrity of cone outer segment tips line with retinal sensitivity after half-dose photodynamic therapy for chronic central serous chorioretinopathy. Am J Ophthalmol. 2012;154(3):579-585.

25. Davies NP, Morland AB. Color Matching in Diabetes: Optical Density of the Crystalline Lens and Macular Pigments. Invest Ophthalmol Vis Sci. 2002;43(1):281-289. 